\title{
A unified conceptual framework for metabolic phenotyping in diagnosis and prognosis
}

Jeremy R. Everett ${ }^{1 *}$, Elaine Holmes ${ }^{2,3}$, Kirill A. Veselkov², John C. Lindon ${ }^{2}$, and Jeremy K. Nicholson ${ }^{2,3 *}$

${ }^{1}$ Medway Metabonomics Research Group, University of Greenwich, Medway Campus, Central Avenue, Chatham Maritime, Kent ME4 4TB UK

${ }^{2}$ Division of Computational and Systems Medicine and MRC-NIHR National Phenome Centre, Department of Surgery and Cancer, Faculty of Medicine, Imperial College London, Sir Alexander Fleming Building, South Kensington, London SW7 2AZ UK

${ }^{3}$ Current address: Australian National Phenome Centre, Murdoch University, Perth WA 6150, Australia * Authors to whom correspondence should be addressed:

j.r.everett@greenwich.ac.uk and Jeremy.Nicholson@murdoch.edu.au

\section{Keywords}

metabonomics, metabolomics, metabotypes, metabolic entropy, systems medicine, personalised medicine, pharmacometabonomics, precision medicine 


\section{Abstract}

Understanding metabotype (multicomponent metabolic characteristics) variation can help generate new diagnostic and prognostic biomarkers and models with the potential to impact patient management. Here we present a suite of conceptual approaches for the generation, analysis and understanding of metabotypes from body fluids and tissues. We describe and exemplify four fundamental approaches to the generation and utilization of metabotype data via multiparametric measurement of: i) metabolite levels; ii) metabolic trajectories; iii) metabolic entropies and iv) metabolic networks and correlations in space and time. This conceptual framework can underpin metabotyping in the scenario of personalised medicine, with the aim of improving clinical outcomes for patients, but it will have value and utility in all areas of metabolic profiling well beyond this exemplar.

\section{Basic concepts of metabolic phenotyping}

In the broadest sense, the phenotype (see Glossary) of an organism refers to the quantitative or qualitative measurement of specific parameters or traits that characterise individual functional biological classes. It is now recognised that the phenotype represents the end product of a complex set of interactions between the genes and environment of an organism, including contributions from the diet, drugs, ageing, exercise, physiological and psychological stressors and the activity of the organisms within us including bacteria, viruses, fungi and parasites. Comprehensive metabolic profiles obtained from individuals are an integral part of the richness of a phenotypic description.

Metabolic phenotyping offers comprehensive molecular structure and concentration information on thousands of compounds found in biological fluids and cell extracts $[1,2]$ in a single analytical run, to generate a metabotype representing the totality of the metabolic state of a particular compartment (biofluid or tissue) of an individual at that point in time. Conceptually, this type of work differs from targeted or univariate metabolic measurements by the notion that the intrinsic, multicomponent patterns of biological systems, and their change in response to disease, treatment, or any intervention, carry uniquely powerful diagnostic information.[1] This approach was subsequently described as "metabonomics" and defined in an experimental, interventional, systems biology framework for understanding the responses of complex systems through time to various interventions or 
pathophysiological stimuli.[2-4] The term metabolomics is also in wide usage and was originally defined in a contrasting observational fashion.[5] However, the analytical methods and approaches are highly convergent and the terms are now used interchangeably. Hereafter, we use the term metabolic phenotyping throughout to describe static and dynamic multiparametric metabolic features.[6] This metabolic phenotyping approach in humans and other mammalian systems has been applied to study drug toxicity,[7] drug metabolism,[8] disease diagnosis, $[9,10]$ the environmental impact of chemicals[11] and nutrition[12]. Note that we distinguish between compounds formed by enzymatic catalysis: metabolites, from those formed by chemical reactions: metabonates.

By defining the set of biochemicals or biomarkers that are characteristic of phenotypes relating to specific physiological or pathological states, one can then infer mechanistic knowledge about those states. Once validated, these metabolic biomarker panels can then be developed into diagnostic or prognostic tools.

Prior to the expression of overt disease, when there is no macroscopic pathology, it can be assumed that there is some degree of metabolic dysregulation. Since the metabotype is based on multiple physical and chemical descriptors, it is likely that it carries latent physiological or phenotypic information relevant to later development of disease.[13] This principle of phenotypic latency enabled the formulation of the concept of pharmacometabonomics, also sometimes known as pharmacometabolomics, where preinterventional metabolic signatures can be used prognostically to predict postinterventional outcomes.[14]

The current status of metabolic profiling in clinical practice is summarised in Box 1.

\section{Metabolite origins}

The metabolites identified in studies of human and animal biofluids can originate from a number of distinct sources. These are broadly classified into three major groups: (i) metabolites that are produced by host cells under host genomic control, (ii) those produced by the enzymes of microorganisms and parasites living within or on the host organism in the microbiome and parasitome respectively, and finally, (iii) metabolites that are produced by processing of environmental agents such as food, drinks, drugs, pollutants etc [23] (Figure 1). 
The complex array of chemicals within the human body thus derives from a combination of human metabolism, exogenous sources and the large number of symbiotic, and sometimes parasitic, organisms residing within the body. As a result, humans can be regarded as superorganisms[15] whose health status is determined by much more than their genes. Gut microorganisms can strongly influence the metabotypes (metabolic phenotypes) of humans and animals with extensive trans-genomic co-metabolism in some compound classes, e.g. bile acids, where certain substances can only be formed via the interactions of human and bacterial genomes. $[13,15,16]$ Studies from multiple groups have shown the microbiome has a role in many diseases including gastrointestinal disorders, $[17,18]$ cancers and efficacy of cancer treatments,[19] metabolic syndrome and obesity[20] and a range of neuropathologies including Alzheimer's disease and autism.[21,22] The microbe-host connections in all these conditions are mediated via complex metabolic and immunological signalling mechanisms that are still poorly understood. The microbiome also impacts heavily on patient responses to therapeutic treatments and so must be considered to be an essential component of future personalised medicine going forward.[23]

The metabolome of biofluids such as urine, plasma or fecal extracts can provide a window on many of these complex trans-genomic interactions. In particular, metabolic phenotyping provides a powerful methodology for studying gut microbial metabolism and the metabolic interactions between the gut bacteria and the host, as well as the status of host metabolism and the influences of diet and the exposome.[12,24] Whilst high throughput genomic sequencing of the microbiome (metagenomics) can provide information on which bacteria are present, it is the metabolic profiles that yield insights on the microbial metabolic functionality. In particular, modulation of microbial-mammalian co-metabolites has been demonstrated in response to obesity,[25] Roux-en-Y gastric bypass surgery[26] and a high fat diet.[27] The gut and other microbiomes therefore represent a target for future therapeutic development and drug targeting.

\section{Biological sample types}

Different biological sample types intrinsically report on the different biological compartments within a complex organism and the metabolites observed will be structurally diverse and the metabolic pathways could be highly topographically distinct. For example, the analysis of metabolites in human breath, will mainly measure the low molecular weight, 
volatile organic compounds derived either from lung tissue and its microbiome[28] in air exchange processes within the lungs and upper respiratory tract of the subject, or directly from exposome volatiles. In direct contrast, samples of human blood plasma will contain non-volatile, low molecular weight metabolites such as simple sugars, hormones, osmolytes and amino acids, as well as macromolecules such as proteins and lipids, supramolecular complexes such as lipoproteins in addition to trace metabolites from the exposome; these metabolites being representative of a particular moment at the time of sampling. Alternatively, analysis of urine will provide information on low molecular weight metabolites derived from the host metabolism, diet, drugs, the exposome and non-human organisms such as gut bacteria; this is a dynamic window reflecting time-averaged metabolism over several hours. The metabolic window on an individual given by different human biological samples will thus be completely different in terms of metabolite type (structural class, molecular weight range, polarity etc), function and origin and will report on different compartments within the subject, some of which will be in metabolic exchange with one another.

In addition to considering which samples to investigate and which analytical technologies to use (see Supplementary Information) to solve the biological or clinical question at hand, it is equally important to consider the sampling methodology. Whilst there are well-documented procedures for sample preparation for both NMR-[29,30] and MS-based metabolic phenotyping approaches,[31],[32] it is now well known that diet, drug-taking and diurnal variation,[33] as well as factors such as gender and age, will all affect the metabolite profiles obtained and several statistical methods have been developed accordingly to correct for such systematic confounding variation, so as to focus on the key clinical and biological questions.[34]

\section{A comprehensive conceptual framework for metabolic phenotyping}

The range of approaches that can be taken in metabolic phenotyping is now so great that it is useful to advance a general conceptual framework for the subject. Thus, we will consider the fundamentally different types of experiments that can be executed for diagnostic and prognostic outcomes and then discuss the various measurement approaches that can be taken in those experiments. 
There are two main approaches to metabolic phenotyping in human and other studies, such as in animals, cellular systems or plants. The first approach comprises interventional or event-based studies where, for example, the differences between control and disease subjects or differential disease diagnoses are required, or the efficacy or otherwise of a therapeutic or other type of intervention is monitored. These can be termed "event interpretation" or "diagnostic studies" (Figure 2, top panel). Here, metabolite metrics are measured before and after the event or treatment e.g. genetic change, physiological or pathological stimulus or other intervention such as drug therapy, and the resulting changes are interpreted in the context of that specific intervention.

The second approach consists of baseline-, or pre-intervention-based studies where the outcome of a treatment or intervention is predicted from a baseline (pre-intervention) study of metabolism, for example, to predict the efficacy of a future therapy regime in groups of subjects. A related type of baseline study is the determination of disease risk factors based upon study of the metabolic composition of biofluids obtained from epidemiological or biobank studies in free-living subjects. These studies can all be termed "event prediction" or "prognostic studies". In a predictive metabolic phenotyping experiment, metabolite metrics are measured for a cohort of individuals prior to an intervention of some kind. The differential, pre-intervention metabolite profiles, or a mathematical model based on those differential profiles, are then used to predict the differential outcomes of the intervention amongst the different subsets of individuals in the cohort (Figure 2, bottom panel). If the intervention is merely the passage of time, then this methodology can be used to predict disease onset in subsets of individuals, sometimes years before its occurrence.

Predictive metabolic phenotyping has its roots in pharmacometabonomics, [14] which is the specific case where the intervention is drug treatment and the prediction is of drug outcome in terms of differential drug pharmacokinetics (PK), metabolism, safety or efficacy amongst the subjects in the cohort, with the prediction made on the basis of an analysis of the differential pre-dose metabolic profiles in that same cohort. This was demonstrated first by the prediction of metabolism and toxicology from pre-intervention urines in a cohort of rats subsequently administered paracetamol.[14] It was later shown that prediction of drug metabolism could also be achieved in a cohort of human volunteers who were administered 
a normal clinical dose of paracetamol.[35] Pharmacometabonomics is thus the metabolic equivalent of pharmacogenomics.[36] Many publications on pharmacometabonomics have now appeared, demonstrating the prediction of drug PK, metabolism, safety and efficacy in animals and humans and even the prediction of survivability in liver disease.[37] The field has recently been reviewed.[36,38] New developments include the powerful combination of technologies utilised in pharmacometabonomics-enabled pharmacogenomics.[39] Given the challenges facing approaches based solely on pharmacogenomics,[40] and the difficulties of predicting phenotypic changes in the presence of complex genome-microbiome interactions, we expect pharmacometabonomics to impact strongly on personalised healthcare in the future.

Predictive metabolic phenotyping in the more general sense has been realised recently in a number of studies, including the large studies by Wang[41] and Wang-Sattler et al[42] who demonstrated prediction of the development of diabetes. In these studies, the intervention is the passage of time, and the baseline metabolite profiles were found to be predictive of disease onset. More recent studies have demonstrated the use of the method for clinical prognosis of cardiovascular events and stroke.[43-45] This type of predictive analysis could also have benefits for personalized healthcare in the future, in terms of tailoring treatments and counseling to those individuals predicted to be most at risk from future disease onset.

Here we also propose a new type of experiment - dynamic predictive metabolic phenotyping. In this approach variant, a cohort of individuals is subjected to a physiological challenge of some type and the differential dynamic responses of the individuals are monitored and used to predict the individual responses of the subjects to a future intervention, which may be related to the initial challenge. The purpose of this is to liberate the latent metabolic phenotype that might not be directly observable under steady-state, non-challenge conditions. The method can also be regarded as the multivariate metabolic equivalent of the biochemical challenge tests[46] such as the glucose tolerance test, that have been used for many years to predict for diabetes. Recently Morris et al.[47] showed that an oral glucose tolerance test could be used to differentiate the metabotypes of a cohort of 214 individuals who clustered into 4 sub-groups, one of which was predicted to be an 'at-risk' cohort. 
Two further variants of predictive metabolic phenotyping approaches are worthy of mention. Firstly, early-onset predictive metabolic phenotyping is a variant where the prediction of intervention outcome is made soon after the intervention (but not before, as in a true predictive experiment) on the basis of early changes to metabolite metrics). This approach was adopted by Winnike et al[48] in a study of the prediction of the safety of paracetamol, after administration of a high dose of the drug to 71 male and female volunteers. Pre-dose biofluid analysis failed to achieve prediction, but metabolite profiles obtained soon after dosing were able to successfully predict reversible liver injury, well before any changes in classical liver damage markers (ALT levels) were seen.

A second variant of predictive metabonomics is longitudinal predictive metabonomic phenotyping, or longitudinal pharmacometabonomics, for the case where the prediction is of the effect of drug treatment. In this variant a cohort of subjects, for example patients, is monitored over time and their metabolic trajectories over that time period are used to phenotype or stratify the patients with respect to optimising their future treatment.[49]

Metabolic phenotyping experiments are often categorised according to whether they are 'targeted' at the analysis of a specific subset of metabolites, or whether they are 'untargeted' with no specific metabolites in mind at the commencement of the study and, as such these latter studies are both agnostic and exploratory.[50] In targeted studies, the subset of metabolites of interest will usually be quantitated to a greater degree than in untargeted studies, perhaps even using internal standards for greater accuracy of metabolite level measurement. The differences between these two approaches are quantitative and technical rather than qualitative and fundamental, as the same measurements e.g. metabolite levels and the same experimental approaches, e.g. event interpretation are typically used in both categories. However, in untargeted studies, multivariate statistical analysis methods will typically be used in order to find patterns in the data and to define statistically significant biomarkers.

\section{Four measurement methodologies for metabolic phenotyping studies}

Once the sample type, sampling protocol, analysis technology and the experimental approach are decided, a key consideration in any metabolic phenotyping experiment is how 
metabolic responses are measured. There are four fundamental ways of measuring and modelling metabolic responses and each will be considered in turn below.

Metabolite concentration measurements. By far the majority of metabolic phenotyping experiments are conducted by measurement of metabolite levels (concentrations) before and after an intervention, which could be, for example, drug treatment of a patient suffering from a disease. It should be noted that metabolite concentrations in urine depend on urinary volumes and should ideally be corrected to excretion rates/kg body mass/24 hours. Changes in metabolite levels can then be correlated with important outcomes associated with the particular intervention e.g. patient recovery from disease following drug treatment. Key metabolite level changes that correlate significantly with clinical outcomes may give rise to the use of those metabolites as metabolic biomarkers for that clinical outcome (Figure $3 \mathrm{~A}$ ). These biomarkers can be mapped to metabolic pathways, potentially giving information on underlying disease mechanisms.

Metabolic trajectory measurements through time. A second approach to the measurement of metabolic responses is to measure metabolite trajectories over time, in response to an intervention and then any recovery from that intervention (Figure 3B) $[51,52,53]$.

The distinction here is that instead of, for example, making two, simple, pre- and postintervention metabolite level measurements in a diagnostic/ event interpretation experiment, a series of measurements are made from a pre-intervention state, through the time period of the intervention and then into the response and recovery period after the intervention. Following the trajectories of individual metabolites, groups of key metabolites, or all observable metabolites in this fashion over time, gives more information on the responses of subjects to an intervention (Figure 3B). For instance, in a recent pre-clinical study on the toxicity of isoniazid, significantly different trajectories were observed between animals that correlated with different clinical outcomes.[52] This approach has also found value in human studies.[54] For cellular studies in particular, using metabolites containing stable isotopes (e.g. ${ }^{13} \mathrm{C}$ for NMR spectroscopy) to give detailed measurements of the rates and routes of incorporation of the label into biochemical pathways has been termed fluxomics.[55-57] Whilst pathway analysis is enabled by fluxomic approaches, it is currently difficult to extend these analyses to the whole body level due to the ensuing high complexity. 
Metabolic statistical entropy measurements. This is a relatively new concept [56] and represents another fundamental way to measure and analyse metabolic phenotyping data. The entropy of a system describes its degree of disorder. Healthy organisms are typically quite ordered by virtue of the existence of control systems that maintain metabolic homeostasis i.e. metabolite levels in a healthy subject in a constant environment (taking into account diurnal variation, menstrual cycle, food and drink intake etc.) will typically not vary significantly. However, in a disease state, or as a result of an intervention of some kind, the metabolic control systems may not fully function, or may be unable to cope with the degree of perturbation to the organism. In such cases, the metabolic entropy of the subject may increase. There are two approaches to the measurement of the entropy of a system and these are based on either a thermodynamic or a configurational freedom approach. These approaches were used in the first exemplification of the method.[58] The thermodynamic approach calculates the non-mechanical work needed to regain metabolic homeostasis. The configurational entropy approach is concerned with the number of metabolic states (i.e. the possible numbers of different levels of metabolites) that are occupied by an individual organism or by a group of different individuals. In the first exemplification by Veselkov et al[58] configurational entropy was defined by a function based on the sum of the pairwise distances in metabolic space between all of the subjects in a particular sample set (see both panels of Figure 3C for an example). Given its unfamiliarity, a new theoretical formulation of metabolic entropy is given in the Supplementary Material (see 'Formulation of metabolic entropy approaches').

Metabolic dependencies, correlations and networks. In any biological system subject to a perturbation or intervention, there will be a cascade of events consequent upon the perturbation and some degree of metabolic disorder will follow. The levels of some metabolites will rise and others will fall. For metabolites that share a biological compartment, or are in exchanging compartments, and that are also part of a biochemical pathway, changes in the level of one metabolite are likely to affect the levels of other metabolites via changes in metabolic flux and regulatory feedback mechanisms. In these circumstances there will be both dependencies and correlations of the levels, trajectories and entropies of one metabolite on others. The ability to determine these dependencies and correlations represents the fourth fashion in which metabolic data can be analysed and 
has been facilitated by recent developments in statistical spectroscopy methods (see Figure 3D for an example relating to interdependency of drug metabolite levels).[34] A good example is the close correlation found between urinary levels of 4-cresylsulphate and phenylacetylglutamine, due to commonality in the microbial transformations of tyrosine and phenylalanine respectively in the early parts of their biosynthetic pathways.[35] The added value of this approach is that it is capable of giving understanding of the biochemical consequences of the intervention applied to the system.[59]

\section{Concluding remarks and future perspectives}

Metabolic phenotyping as a formally-defined scientific endeavour has made significant impact in terms of understanding human and animal disease states from an integrated systems biology perspective.[60] Progress has been very rapid recently; in particular, the development of methods enabling the prediction of effects in animals and humans based on pre-dose metabolic profiles[14,35,41,42]. In the light of these significant recent developments, the new, unified framework presented here for the different fundamental approaches to metabolic phenotyping experiments (diagnostic and prognostic/predictive) and the four fundamental approaches to metabolite measurements (concentrations, trajectories, entropies and networks/correlations) will help place these and future experiments in an appropriate context.

Predictive metabolic phenotyping in general and pharmacometabonomics in particular are not expected to replace other predictive sciences such as pharmacogenomics or pharmacometagenomics, but it is likely that an optimized combination of both genomic and metabolic approaches will find application for understanding inter-patient phenotypic differences.[61,62] One outstanding question is what proportion of phenotypic variance will be explained by genomic relative to metabolic approaches (see Outstanding Questions). For example, the differences between cancer patients who respond to a drug treatment versus those who do not, or perhaps more strikingly, the differences between mice of two genotypes, where complex genome - microbiome interactions may complicate simple genetic interpretations. We do not underestimate the degree of difficulty involved in implementing such stratified medicine paradigms in modern healthcare. However, the rewards in terms of optimized treatment of patients, reduced morbidity and mortality, and improved health economics make this a worthwhile challenge. Ultimately, systems medicine 
has to be practical in the real world and deliver clinically-actionable outcomes: the concept of patient journey phenotyping[63] implemented using metabolic tools will greatly assist this. One excellent, recent, surgical implementation of metabolic profiling is that of the iknife, which analyses the compounds present in the smoke created by surgical cauterisation of patient tissue, using an MS approach. This technology[64-66] allows the surgeon to distinguish metabolically, and in real-time, the type of tissue being cut. The i-knife is currently in clinical trials e.g. the REI-EXCISE iKnife Study (Clinical Trial Numberi: NCT03432429) and in the process of being commercialised.

More recently, the linkage of genetic and metabolic information has gained force and analysis of large sample databases from bio-banks and epidemiology studies has shown much promise.[67] This has led to genetic-metabolic correlation studies providing substantial systems biology integration, and in the case of epidemiology, the development of the metabolome-wide association study (MWAS)[68] approach, analogous to the widely-used genome-wide association study (GWAS) approach.

To make progress in the above strategic areas will require new analytical techniques, new innovative means for data processing and visualization, as well as new approaches to integrate these innovations into clinical practice. Given the emerging understanding of humans as super-organisms[15] and the impact of the microbiome[23] upon health, disease, and treatments, new, rapid methods for microbiological characterization are also required (see Outstanding Questions).

Furthermore, the establishment of large, representative, long-term, sample bio-banks and dedicated Phenome Centres such as those in London, Birmingham[6] and Singapore ii will facilitate the use of metabotypes for clinical assessment. This will produce novel metabolic insights, improved biomarkers and a rich resource for analysis by future technologies and algorithms. The framework and approaches discussed here can then be extended into many areas of clinical medicine and personalised medicine for future patient benefit. 


\section{Acknowledgements}

We acknowledge the many contributions from our colleagues in metabolic research over the past four decades and in particular Professor lan Wilson, Professor Zoltan Takats, and the staff of the MRC-NHR National Phenome Centre (established by a grant from the UK Medical Research Council and National Institute of Health Research).

\section{Resources:}

i) https://clinicaltrials.gov/

ii) http://www.lkcmedicine.ntu.edu.sg/Research/Singapore-PhenomeCentre/Pages/Singapore-Phenome-Centre.aspx

\section{References}

1. Nicholson JK, Wilson ID. High-Resolution Proton Magnetic Resonance Spectroscopy of Biological Fluids. Progress in nuclear magnetic resonance spectroscopy 1989; 21: 449-501

2. Nicholson JK, Lindon JC, Holmes E. 'Metabonomics': understanding the metabolic responses of living systems to pathophysiological stimuli via multivariate statistical analysis of biological NMR spectroscopic data. Xenobiotica 1999; 29: 1181-1189

3. Nicholson JK, Connelly J, Lindon JC et al. Metabonomics: a platform for studying drug toxicity and gene function. Nat Rev Drug Discov 2002; 1: 153-161

4. Lindon J, Nicholson J, Holmes E et al. Metabonomics: Metabolic processes studied by NMR spectroscopy of biofluids. Concepts in Magnetic Resonance 2000; 12: 289-320

5. Fiehn O. Metabolomics--the link between genotypes and phenotypes. Plant Mol Biol 2002; 48: 155-171

6. Nicholson JK, Darzi A, Holmes E et al. Metabolic phenotyping in personalized and public healthcare. London: Academic Press; 2016

7. Lindon JC, Keun HC, Ebbels TM et al. The Consortium for Metabonomic Toxicology (COMET): aims, activities and achievements. Pharmacogenomics 2005; 6: 691-699

8. Waters NJ. The Role of Metabonomics at the Interface Between Drug Metabolism and Safety Assessment. Current Drug Metabolism 2010; 11: 686-692

9. Moolenaar SH, Engelke UFH, Wevers RA. Proton nuclear magnetic resonance spectroscopy of body fluids in the field of inborn errors of metabolism. Annals of Clinical Biochemistry 2003; 40: $16-24$

10. Emwas A-HM, Salek RM, Griffin JL et al. NMR-based metabolomics in human disease diagnosis: applications, limitations, and recommendations. Metabolomics 2013; 9: 10481072

11. Bonvallot $\mathrm{N}$, Tremblay-Franco $\mathrm{M}$, Chevrier $\mathrm{C}$ et al. Potential Input From Metabolomics for Exploring and Understanding the Links Between Environment and Health. Journal of Toxicology and Environmental Health-Part B-Critical Reviews 2014; 17: 21-44

12. Garcia-Perez I, Posma JM, Gibson R et al. Objective assessment of dietary patterns by use of metabolic phenotyping: a randomised, controlled, crossover trial. Lancet Diabetes Endocrinol 2017; 5: 184-195

13. Nicholson JK, Wilson ID. Understanding 'global' systems biology: Metabonomics and the continuum of metabolism. Nature Reviews Drug Discovery 2003; 2: 668-676 
14. Clayton T, Lindon J, Cloarec $\mathrm{O}$ et al. Pharmaco-metabonomic phenotyping and personalized drug treatment. Nature 2006; 440: 1073-1077

15. Nicholson JK, Holmes E, Lindon JC et al. The challenges of modeling mammalian biocomplexity. Nature Biotechnology 2004; 22: 1268-1274

16. Nicholson JK, Holmes E, Wilson ID. Gut microorganisms, mammalian metabolism and personalized health care. Nature Reviews Microbiology 2005; 3: 431-438

17. Ahmad OF, Akbar A. Microbiome, antibiotics and irritable bowel syndrome. British Medical Bulletin 2016; 120: 91-99

18. Rogers GB, Narkewicz MR, Hoffman LR. The CF gastrointestinal microbiome: Structure and clinical impact. Pediatric Pulmonology 2016; 51: S35-S44

19. Contreras AV, Cocom-Chan B, Hernandez-Montes $\mathrm{G}$ et al. Host-Microbiome Interaction and Cancer: Potential Application in Precision Medicine. Frontiers in Physiology 2016; 7:

20. Tang WHW, Hazen SL. The Gut Microbiome and Its Role in Cardiovascular Diseases. Circulation 2017; 135: 1008-1010

21. Yap IKS, Angley M, Veselkov KA et al. Urinary Metabolic Phenotyping Differentiates Children with Autism from Their Unaffected Siblings and Age-Matched Controls. Journal of Proteome Research 2010; 9: 2996-3004

22. Sharon G, Sampson TR, Geschwind DH et al. The Central Nervous System and the Gut Microbiome. Cell 2016; 167: 915-932

23. Kochhar Se, Martin F-Pe. Metabonomics and gut microbiota in nutrition and disease: Springer; 2015

24. Rappaport SM, Barupal DK, Wishart D et al. The Blood Exposome and Its Role in Discovering Causes of Disease. Environmental Health Perspectives 2014; 122: 769-774

25. Xie G, Ma X, Zhao A et al. The Metabolite Profiles of the Obese Population Are GenderDependent. Journal of Proteome Research 2014; 13: 4062-4073

26. Wu Q, Li JV, Seyfried F et al. Metabolic phenotype-microRNA data fusion analysis of the systemic consequences of Roux-en-Y gastric bypass surgery. International Journal of Obesity 2015; 39: 1126-1134

27. Walker A, Pfitzner B, Neschen $S$ et al. Distinct signatures of host-microbial metametabolome and gut microbiome in two C57BL/6 strains under high-fat diet. Isme Journal 2014; 8: 2380-2396

28. Twomey KB, Alston M, An SQ et al. Microbiota and Metabolite Profiling Reveal Specific Alterations in Bacterial Community Structure and Environment in the Cystic Fibrosis Airway during Exacerbation. Plos One 2013; 8:

29. Beckonert O, Keun HC, Ebbels TMD et al. Metabolic profiling, metabolomic and metabonomic procedures for NMR spectroscopy of urine, plasma, serum and tissue extracts. Nature Protocols 2007; 2: 2692-2703

30. Dona AC, Kyriakides M, Scott F et al. A guide to the identification of metabolites in NMRbased metabonomics/metabolomics experiments. Computational and Structural Biotechnology Journal 2016; 14: 135-153

31. Gika H, Theodoridis G. Sample preparation prior to the LC-MS-based metabolomics/metabonomics of blood-derived samples. Bioanalysis 2011; 3: 1647-1661

32. Want EJ, Wilson ID, Gika $\mathrm{H}$ et al. Global metabolic profiling procedures for urine using UPLCMS. Nat Protoc 2010; 5: 1005-1018

33. Giskeødegård GF, Davies SK, Revell VL et al. Diurnal rhythms in the human urine metabolome during sleep and total sleep deprivation. Sci Rep 2015; 5: 14843

34. Robinette SL, Lindon JC, Nicholson JK. Statistical Spectroscopic Tools for Biomarker Discovery and Systems Medicine. Analytical Chemistry 2013; 85: 5297-5303

35. Clayton TA, Baker D, Lindon JC et al. Pharmacometabonomic identification of a significant host-microbiome metabolic interaction affecting human drug metabolism. Proceedings of the National Academy of Sciences of the United States of America 2009; 106: 14728-14733 
36. Everett JR. Pharmacometabonomics in humans: a new tool for personalized medicine. Pharmacogenomics 2015; 16: 737-754

37. McPhail MJW, Shawcross DL, Lewis MR et al. Multivariate metabotyping of plasma predicts survival in patients with decompensated cirrhosis. Journal of Hepatology 2016; 64: 10581067

38. Everett JR. From Metabonomics to Pharmacometabonomics: The Role of Metabolic Profiling in Personalized Medicine. Frontiers in Pharmacology 2016; 7: 15

39. Ji Y, Hebbring S, Zhu H et al. Glycine and a glycine dehydrogenase (GLDC) SNP as citalopram/escitalopram response biomarkers in depression: pharmacometabolomicsinformed pharmacogenomics. Clin Pharmacol Ther 2011; 89: 97-104

40. Kitsios GD, Kent DM. Personalised medicine: not just in our genes. British Medical Journal 2012; 344:

41. Wang TJ, Larson MG, Vasan RS et al. Metabolite profiles and the risk of developing diabetes. Nature Medicine 2011; 17: 448-453

42. Wang-Sattler R, Yu Z, Herder C et al. Novel biomarkers for pre-diabetes identified by metabolomics. Molecular Systems Biology 2012; 8:

43. Shah SH, Sun J-L, Stevens RD et al. Baseline metabolomic profiles predict cardiovascular events in patients at risk for coronary artery disease. American Heart Journal 2012; 163: 844$+$

44. Rizza S, Copetti M, Rossi C et al. Metabolomics signature improves the prediction of cardiovascular events in elderly subjects. Atherosclerosis 2014; 232: 260-264

45. Jove M, Mauri-Capdevila G, Suarez I et al. Metabolomics predicts stroke recurrence after transient ischemic attack. Neurology 2015; 84: 36-45

46. Vis DJ, Westerhuis JA, Jacobs DM et al. Analyzing metabolomics-based challenge tests. Metabolomics 2015; 11: 50-63

47. Morris C, O'Grada C, Ryan M et al. Identification of Differential Responses to an Oral Glucose Tolerance Test in Healthy Adults. Plos One 2013; 8:

48. Winnike JH, Li Z, Wright FA et al. Use of pharmaco-metabonomics for early prediction of acetaminophen-induced hepatotoxicity in humans. Clin Pharmacol Ther 2010; 88: 45-51

49. Nicholson JK, Everett JR, Lindon JC. Longitudinal pharmacometabonomics for predicting patient responses to therapy: drug metabolism, toxicity and efficacy. Expert Opin Drug Metab Toxicol 2012; 8: 135-139

50. Dunn WB, Goodacre R, Neyses $L$ et al. Integration of metabolomics in heart disease and diabetes research: current achievements and future outlook. Bioanalysis 2011; 3: 2205-2222

51. Holmes E, Bonner FW, Sweatman BC et al. Nuclear-Magnetic-Resonance Spectroscopy And Pattern-Recognition Analysis Of The Biochemical Processes Associated With The Progression Of And Recovery From Nephrotoxic Lesions In The Rat Induced By Mercury(li) Chloride And 2-Bromoethanamine. Molecular Pharmacology 1992; 42: 922-930

52. Cunningham K, Claus SP, Lindon JC et al. Pharmacometabonomic Characterization of Xenobiotic and Endogenous Metabolic Phenotypes That Account for Inter-individual Variation in Isoniazid-Induced Toxicological Response. J Proteome Res 2012; 11: 4630-4642

53. Keun HC, Ebbels TM, Bollard ME et al. Geometric trajectory analysis of metabolic responses to toxicity can define treatment specific profiles. Chem Res Toxicol 2004; 17: 579-587

54. Nevedomskaya E, Mayboroda OA, Deelder AM. Cross-platform analysis of longitudinal data in metabolomics. Mol Biosyst 2011; 7: 3214-3222

55. Charidemou E, Ashmore T, Griffin JL. The use of stable isotopes in the study of human pathophysiology. International Journal of Biochemistry \& Cell Biology 2017; 93: 102-109

56. Saborano R, Eraslan Z, Roberts J et al. A framework for tracer-based metabolism in mammalian cells by NMR. Scientific Reports 2019; 9:

57. Basler G, Fernie AR, Nikoloski Z. Advances in metabolic flux analysis toward genome-scale profiling of higher organisms. Bioscience Reports 2018; 38: 
58. Veselkov KA, Pahomov VI, Lindon JC et al. A metabolic entropy approach for measurements of systemic metabolic disruptions in patho-physiological States. J Proteome Res 2010; 9: 3537-3544

59. Frainay C, Jourdan F. Computational methods to identify metabolic sub-networks based on metabolomic profiles. Briefings in Bioinformatics 2017; 18: 43-56

60. Ed. Lindon J, Nicholson, JK and Holmes E. The handbook of metabolic phenotyping. Oxford: Elsevier; 2019

61. Lewis JP, Yerges-Armstrong LM, Ellero-Simatos S et al. Integration of Pharmacometabolomic and Pharmacogenomic Approaches Reveals Novel Insights Into Antiplatelet Therapy. Clinical Pharmacology \& Therapeutics 2013; 94: 570-573

62. Neavin D, Kaddurah-Daouk R, Weinshilboum R. Pharmacometabolomics informs pharmacogenomics. Metabolomics 2016; 12:

63. Kinross JM, Holmes E, Darzi AW et al. Metabolic phenotyping for monitoring surgical patients. Lancet 2011; 377: 1817-1819

64. Balog J, Kumar S, Alexander J et al. In Vivo Endoscopic Tissue Identification by Rapid Evaporative Ionization Mass Spectrometry (REIMS). Angewandte Chemie-International Edition 2015; 54: 11059-11062

65. Alexander J, Gildea L, Balog J et al. A novel methodology for in vivo endoscopic phenotyping of colorectal cancer based on real-time analysis of the mucosal lipidome: a prospective observational study of the iKnife. Surgical Endoscopy and Other Interventional Techniques 2017; 31: 1361-1370

66. Phelps DL, Balog J, Gildea LF et al. The surgical intelligent knife distinguishes normal, borderline and malignant gynaecological tissues using rapid evaporative ionisation mass spectrometry (REIMS). British Journal of Cancer 2018; 118: 1349-1358

67. Gieger C, Geistlinger L, Altmaier E et al. Genetics Meets Metabolomics: A Genome-Wide Association Study of Metabolite Profiles in Human Serum. Plos Genetics 2008; 4:

68. Dumas ME, Wilder SP, Bihoreau MT et al. Direct quantitative trait locus mapping of mammalian metabolic phenotypes in diabetic and normoglycemic rat models. Nat Genet 2007; 39: 666-672

69. Wild CP. Complementing the genome with an "exposome": The outstanding challenge of environmental exposure measurement in molecular epidemiology. Cancer Epidemiology Biomarkers \& Prevention 2005; 14: 1847-1850

70. Song $\mathrm{Z}$, Wang $\mathrm{H}$, Yin $\mathrm{X}$ et al. Application of NMR metabolomics to search for human disease biomarkers in blood. Clinical Chemistry and Laboratory Medicine 2019; 57: 417-441

71. Otvos JD, Guyton JR, Connelly MA et al. Relations of GlycA and lipoprotein particle subspecies with cardiovascular events and mortality: A post hoc analysis of the AIM-HIGH trial. Journal of Clinical Lipidology 2018; 12: 348-355

72. Otvos JD, Jeyarajah EJ, Cromwell WC. Measurement issues related to lipoprotein heterogeneity. American Journal of Cardiology 2002; 90: 22I-291

73. Jimenez B, Holmes E, Heude C et al. Quantitative Lipoprotein Subclass and Low Molecular Weight Metabolite Analysis in Human Serum and Plasma by H-1 NMR Spectroscopy in a Multilaboratory Trial. Analytical Chemistry 2018; 90: 11962-11971

74. Emwas A-H, Luchinat C, Turano P et al. Standardizing the experimental conditions for using urine in NMR-based metabolomic studies with a particular focus on diagnostic studies: a review. Metabolomics 2015; 11: 872-894 


\section{TEXT BOX}

Box 1: Current status of metabolic phenotyping in clinical practice.

The current status of metabolic phenotyping in clinical practice was recently critically reviewed by James Kinross in Chapter 16 of 'The Handbook of Metabolic Phenotyping'[60] and Jiang et al published a comprehensive survey of the recent application of nuclear magnetic resonance (NMR) spectroscopy to identify disease biomarkers in human blood.[70] Whilst initial results are promising in many areas of clinical practice, it was concluded that more validation and greater standardisation of methods is required to create the evidence base for wider adoption of metabolic phenotyping in clinical practice. Ioanna Tzoulaki and colleagues reviewed epidemiological applications of metabolic phenotyping in Chapter 17 of the same book ('The Handbook of Metabolic Phenotyping'),[60] with similar conclusions. One promising clinical application is in the area of human plasma testing for lipoprotein levels, an area pioneered by Otvos et al.[71,72] In this regard, the results of a recent inter-laboratory ring test[73] on the precision of metabolic profiling of human plasma were encouraging. This study[73] demonstrated that NMR methods had internal QC variance lower than that required by the National Cholesterol Education Program (NCEP) criteria for lipid testing and should therefore be suitable for clinical diagnostic purposes. In addition, the 2015 study by Emwas et al[74] highlighted the utility of urine analysis for disease diagnosis and proposed guidelines for standardisation of methods. 


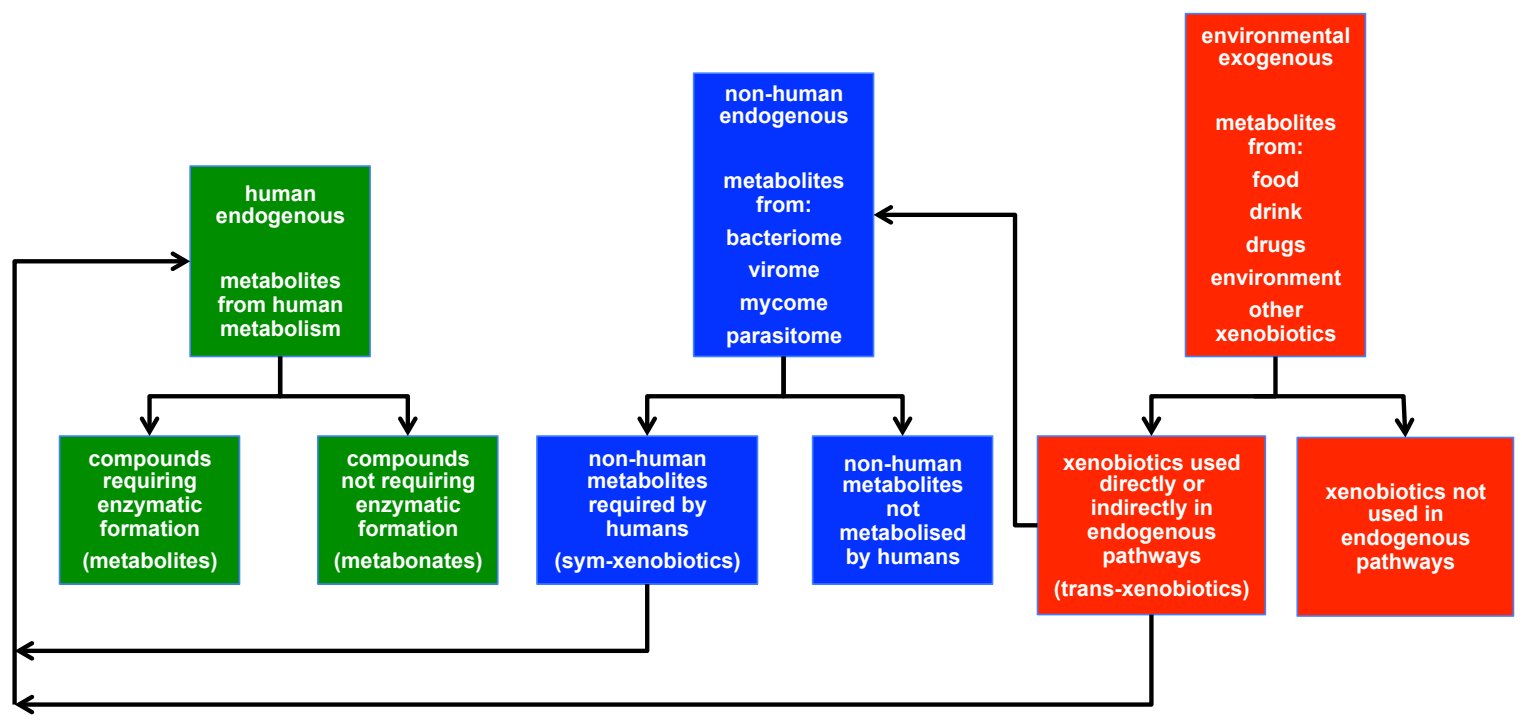

Figure 1. The complex origins and interactions of the metabolites that determine phenotypes. Metabolites can be obtained from three major sources: from the host (human endogenous), from the microbiome (non-human endogenous) and from the environment (exogenous). Sym-xenobiotics are co-metabolites produced by co-metabolism by human and e.g. microbiome pathways. Trans-xenobiotics are xenobiotics that are converted into endogenous metabolites or xenobiotics that are processed directly in endogenous pathways, such as ethanol. 
Metabonomics: Diagnostic or Interventional Approach
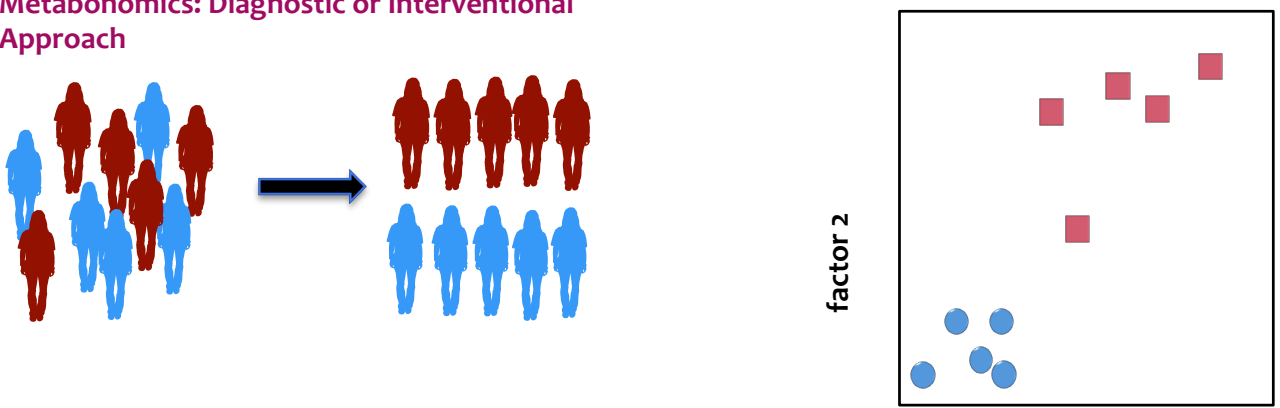

factor 1

Predictive Metabonomics: Prognostic or Predictive Approach

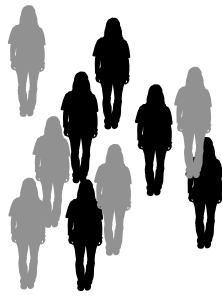

Time A
TIME

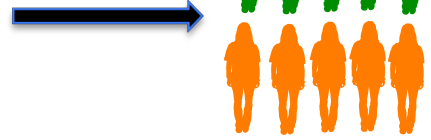

Time B

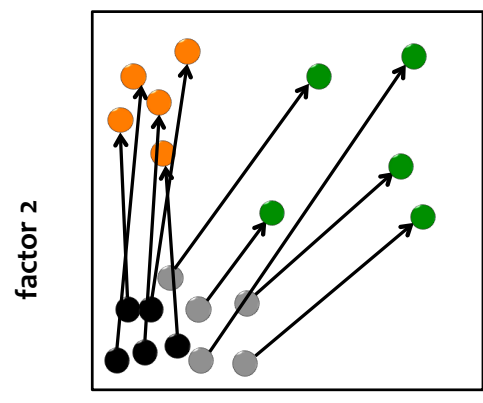

factor 1

Figure 2. Schematic representations of the two key experimental approaches to metabolic phenotyping with subjects on the left-hand side and a multivariate analysis of their metabolite profiles on the right hand side. Top. Classical diagnostic metabonomics approach, differentiating healthy individuals (blue) from subjects with a disease (red) at one given time point. A multivariate principal components (PC) scores plot is on the right. Bottom. Predictive metabonomics approach. In this case there is a difference (although not complete discrimination) in the pre-intervention metabolic profiles at Time A between two sub-groups of the subjects (black and grey). This allows prediction of different postintervention states at Time B for the two sub groups (orange and green circles respectively). The arrows in the PC scores plot connect the metabolic trajectories of each subject in the two sub-groups over the two time points measured, before and after the intervention. 

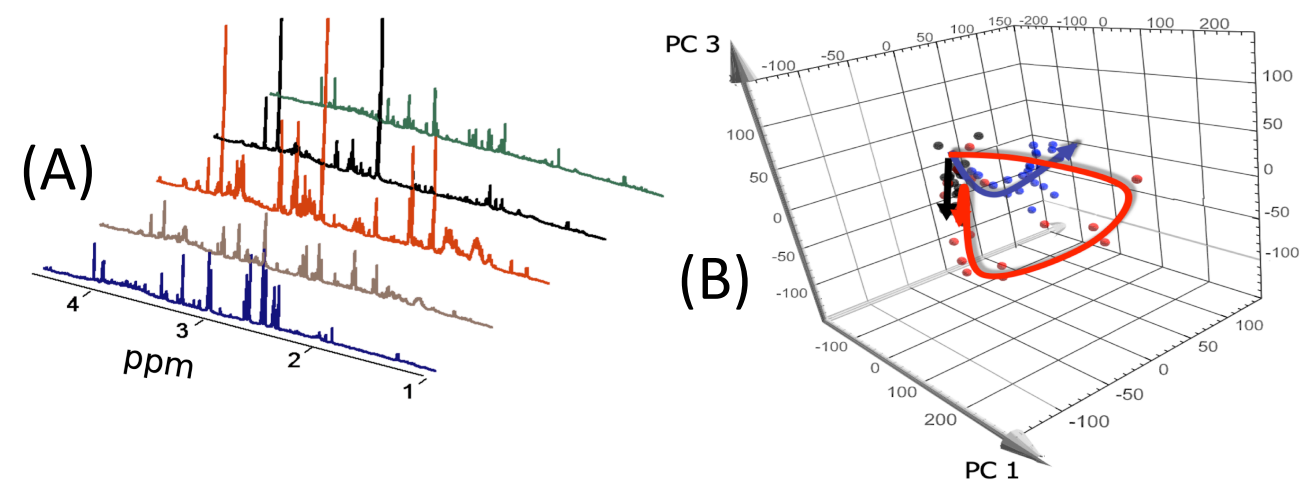

(C)
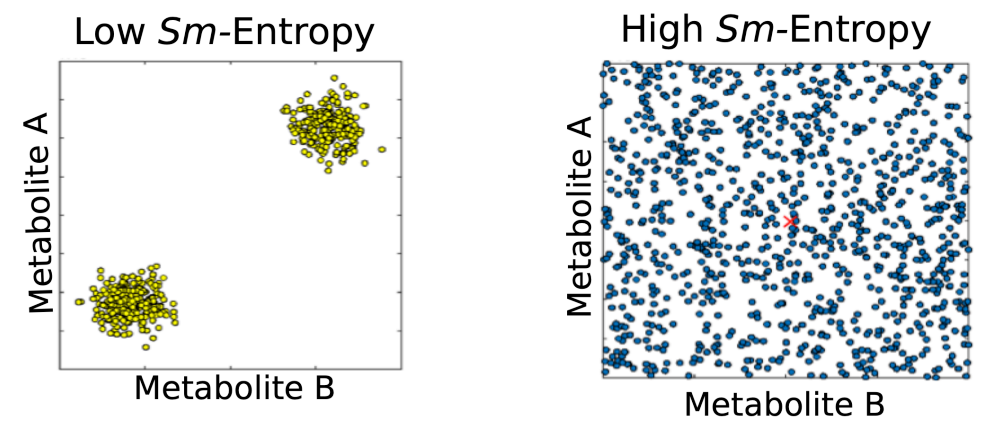

Metabolite B

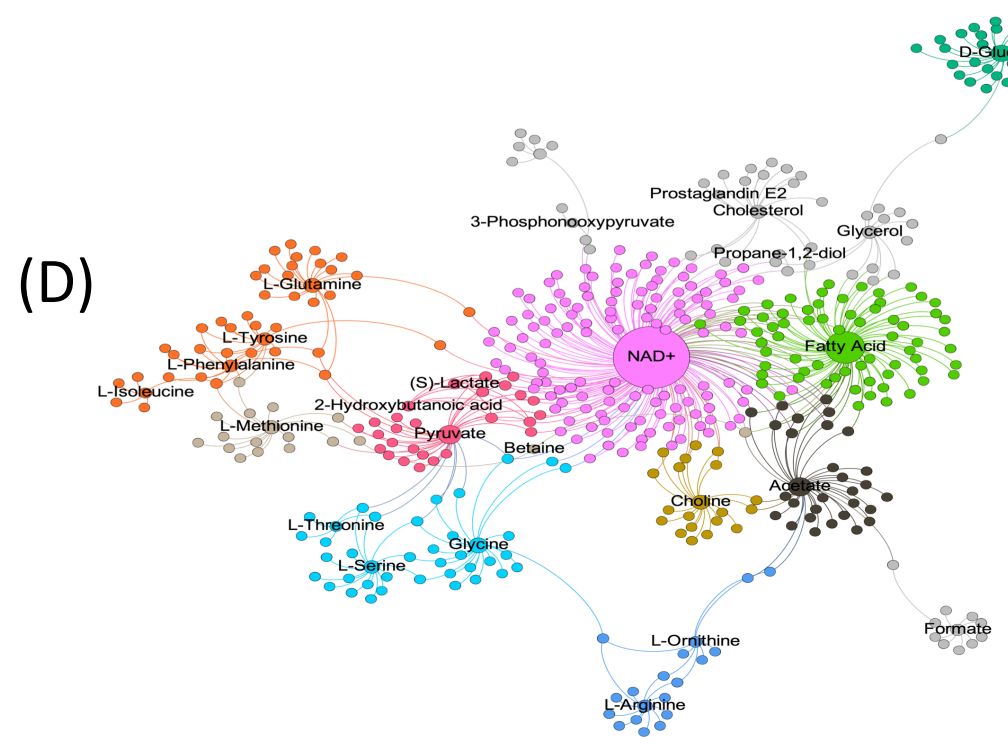

Figure 3. Examples of the four principal approaches to the measurement of metabolic phenotyping data: via (A) metabolite levels, (B) metabolite trajectories, (C) metabolite entropies and (D) metabolite correlations and dependencies.

(A) Metabolite level measurements exemplified by four ${ }^{1} \mathrm{H}-\mathrm{NMR}$ urine spectra of SpragueDawley rats. (B) Metabolic trajectories obtained from principal components (PC) scores of 
time-resolved ${ }^{1} \mathrm{H}-\mathrm{NMR}$ urinary profiles of Sprague-Dawley rats subjected to $50 \%$ caloric restriction intervention (blue), no intervention (control, black) and high-dose of hydrazine toxicity (red). The latter shows deviation of metabolic trajectory due to the hydrazineinduced toxicity effects and subsequent system's recovery to the control behaviour. (C) Metabolic entropy concept demonstrated on simulated "clustered" and "uniformly" distributed levels of two metabolites. The higher the heterogeneity of metabolite levels, the higher the entropy value. Here, both distributions have equal variance but different entropy values showing the advantage of entropic approaches to capture the heterogeneity or disorder in metabolic phenotypes as opposed to the variability. (D) Metabolite networks illustrated with a subset of metabolite-protein interaction networks derived from KEGG/OmicsNet (https://www.omicsnet.ca/). 


\section{Glossary}

diagnosis: the characterisation of an organism, disease state, phenotype or response to an intervention exposome: all those chemicals in the environment to which a human is exposed.[69]

GWAS (genome-wide association study): an observational study that aims to associate a particular biological trait with a genetic variant

MWAS (metabolite-wide association study): an observational study that aims to associate a particular biological trait such as disease state with metabolic variation or phenotype

metabolic biomarker: a structurally-defined metabolite or metabolic feature, verified to be associated with a particular phenotypic, disease, diagnostic or prognostic class and that can be used specifically to identify those classes or states

metabolic entropy: the degree of disorder of metabolite characteristics, such as concentrations, in an individual or in a group of subjects

metabotype: multicomponent metabolic characteristics that result from the cumulative interactions of genetic variation, gene products and environmental exposures and that can be related directly to disease risks and therapeutic responses, also known as the metabolic phenotype

metabolic trajectory: the changes in metabolite concentrations over time in response to an intervention

metabolite: a compound in a biological matrix of an organism that is produced in that organism by an enzymatic pathway

metabolomics: metabolic profiling defined in an observational fashion as 'a comprehensive analysis in which all the metabolites of a biological system are identified and quantified'

metabolome: the full set of metabolites within, or that can be secreted from, a biological system such as a cell type or tissue

metabonate: a compound in a biological matrix of an organism that is produced in that organism by a chemical pathway without enzymatic involvement. This term is also used to describe molecular artifacts produced chemically during sample extraction, isolation, purification or analysis procedures

metabonomics: metabolic profiling defined in an experimental fashion as 'the quantitative measurement of the multiparametric metabolic response of living systems to pathophysiological stimuli or genetic modification'

metabotypes or metabolic phenotypes: are the multicomponent metabolic characteristics that result from the cumulative interactions of genetic variation, gene products and environmental exposures that can be related directly to disease risks and therapeutic responses. The metabotype is a probabilistic, multiparametric description of an organism in a given physiological state based on analysis of its cell types, biofluids and tissues

microbiome: the collection of microorganisms present both in and on an organism, in a variety of environmental niches: the bacteriome, virome and mycobiome refer to the collection of bacteria, viruses and fungi, that constitute the microbiome

MVA (multivariate [statistical] analysis): a method for the analysis of multiple variables in an experiment or observation at a time and the simplification of the analysis problem by reduction of the large number of initial variables to a small number of key factors 
parasitome: the collection of parasites associated with the host organism.

personalized medicine: the use of genomic, molecular and clinical information to select treatments or medicines that are more likely to be both effective and safe for that patient: also known as precision medicine or stratified medicine

pharmacogenomics: the prediction of the effects of a drug on the basis of individual genetic profiles

pharmacometabolomics: this term is used synonymously with pharmacometabonomics (see below), but is sometimes erroneously used to describe the investigation of the effects of a drug on an organism: this is just diagnostic metabonomics

pharmacometabonomics: the prediction of the properties of a drug (efficacy, toxicity, metabolism, pharmacokinetics) on the basis of a mathematical model of pre-dose metabolite profiles

pharmacometagenomics: the prediction of the properties of a drug on the basis of a mathematical model of pre-dose microbiome profiles

phenotype: the quantitative or qualitative measurement of specific parameters or traits that characterise individual functional biological classes or groups

predictive metabolic phenotyping: the prediction of the outcome of an intervention in an individual based on a mathematical model of pre-intervention metabolite profiles. The intervention could be a change in diet, exercise, the passage of time, surgical treatment etc. Pharmacometabonomics is one specific case of predictive metabolic phenotyping in which the intervention is drug treatment

prognosis: the prediction of disease onset, disease outcome or the outcome of an intervention such as drug treatment 